doi:10.13108/2020-12-3-81

\title{
PROPERTIES OF CONVEX HULL GENERATED BY INHOMOGENEOUS POISSON POINT PROCESS
}

\author{
I.M.KHAMDAMOV
}

\begin{abstract}
The paper is devoted to the limit distribution study of the exterior of a convex hull generated by independent observations of two-dimensional random points having Poisson distributions above the parabola. Following P. Groeneboom [1], we note that near the boundary of support, the Binomial point process is almost indistinguishable from the Poisson point process. Therefore, the approximation of a Binomial point process to a Poisson process is not considered here; it is believed that it is sufficient to study the functionals of the convex hull generated by the Poisson point process. Using the modified P. Groeneboom technique, the so-called strong mixing and martingale properties of the vertex Markovian jump stationary process, the asymptotic expressions are obtained for the expectation and variance of the external part of the area of the convex hull inside the parabola. This is a continuation of results by H. Carnal in [2], where an asymptotic expression was found only for mean values of basic functionals of a convex hull. The asymptotic expression for the variance of the area of a convex hull was later obtained by J. Pardon [3] as no regularity conditions were imposed on the boundary of the support of a uniform distribution. The asymptotic expressions obtained here are used in the proofs of the central limit theorem for the area of the convex hull. Similar results were established in the studies by A.J. Cabo and P. Groeneboom [4] for the case as the initial distribution in a convex polygon is uniform.
\end{abstract}

Keywords: convex hull, random points, Poisson point process.

Mathematics Subject Classification: 60F05, 60D05

\section{INTRODUCTION}

Many researchers studied the distribution of boundary functionals of convex hulls. From an analytical point of view, these functionals have a very complicated nature. For many years, due to the lack of appropriate research methods, the main progress in this direction was limited by studying the expectation of a number of vertices, area and perimeter of a random polygon, see [2], [5], [6], [7], [8]). A great progress in this direction for the first time was achieved in [1. Here the approximation methods for binomial point processes with homogeneous Poisson point processes were successfully applied. Then the powerful properties were found, namely, martingality, strong mixing, and stationarity of functionals of the vertex process of a convex hull generated by the Poisson point process. The limit distribution for a number of vertices of a convex hull was obtained in the case, when the support of the initial uniform distribution is either a convex polygon or an ellipse. Then the method was developed for limit theorems for the area and perimeter of a convex hull in a polygon [4] and for the area in a disk [9]. These methods were applied in [10] to prove the limit theorems for the number of vertices, perimeter, and area of the convex hull, for the case when the tails of the initial distribution had an exponential form, including, in particular, the normal distribution. At the same time, using the methods given in [1], by the direct probabilistic method [11] the limiting joint probability

I.M.Khamdamov, Properties of Convex hull generated by inhomogeneous Poisson point PROCESS.

(C) I.M.Khamdamov, 2020.

Поступила 14 январл 20202. 
distribution was obtained for the number of vertices, area and perimeter of a random convex hull generated by uniformly distributed points inside a polygon. In [12], in a one-dimensional case, it was proved that the contribution of the extreme terms of the variational series to the sum was significant when the distribution tail of initial random variable behaved as a regularly varying function. In a multidimensional case, the convex hull is the most correct generalization of the extreme terms of variational series and, therefore, the present study can be considered as a continuation of earlier research by authors.

\section{Formulation of PROBlem AND MAIN RESUltS}

Let the distribution support $A$ be the unit disk centered at a point $(0,1)$. Assume that random points $\left(r_{i}, \alpha_{i}\right)$ are given in the polar coordinate system with the origin at $(0,1)$ in a disk $A$, where $r_{i}$ and $\alpha_{i}$ are independent and $\alpha_{i}$ is uniformly distributed in $[-\pi, \pi]$ and

$$
\mathbb{P}\left(r_{i}>1-x\right)=x^{\beta} L\left(\frac{1}{x}\right), \quad 0<x<1, \quad \beta \geqslant 1,
$$

where $L(x)$ is the slowly varying function in sense of Karamata represented in the following form

$$
L(u)=\exp \left\{\int_{1}^{u} \frac{\varepsilon(t)}{t} d t\right\}, \quad \varepsilon(t) \rightarrow 0, \quad t \rightarrow \infty .
$$

We assume that $X_{i}=r_{i} \sin \alpha_{i}, 1-Y_{i}=r_{i} \cos \alpha_{i}$ and we denote by $C_{n}$ the convex hull generated by these random points $\left(X_{1}, Y_{1}\right) ;\left(X_{2}, Y_{2}\right) ; \ldots ;\left(X_{n}, Y_{n}\right)$. The symbols $\nu_{n}, s_{n}$ and $l_{n}$ stand for the numbers of vertices, area and perimeter of $C_{n}$, respectively. Under such assumptions, asymptotic mean values for the functionals of the convex hull were obtained in [2].

In this paper, we study the functional $s_{n}$ as the random points are distributed in the same way as in (2.1). Following [1],[11], [13]-[15], it is sufficient to study the functionals of the convex hull in the case when it is generated by the Poisson point process. That is, random points with distribution (2.1) are easily approximated by inhomogeneous Poisson point processes $\Pi_{n}(\cdot)$, defined inside a parabola

$$
R_{n}=\left\{(x, y): \frac{x^{2}}{2 b_{n}} \leqslant y\right\}
$$

with intensity

$$
\Lambda_{n}(A)=\left\{\begin{array}{cl}
\frac{1}{2 \pi \sqrt{b_{n}} L\left(b_{n}\right)} \int_{A} \frac{\partial}{\partial y}\left\{\left(y-\frac{x^{2}}{2 b_{n}}\right)^{\beta} L\left(\frac{b_{n}}{y-\frac{x^{2}}{2 b_{n}}}\right)\right\} d x d y & \text { as } \quad A \subset R_{n}, \\
0 & \text { as } \quad A \not \subset R_{n},
\end{array}\right.
$$

where $b_{n}$ is the least root of equation

$$
n b_{n}^{-\left(\beta+\frac{1}{2}\right)} L\left(b_{n}\right)=1
$$

For all $a \in \mathbb{R}$ we define the vertices of process $W_{n}(a)=\left(X_{n}(a), Y_{n}(a)\right)$ as points $\left(X_{i}, Y_{i}\right)$, for which $Y_{i}-a X_{i}$ is minimal. In view of the definition of $W_{n}(a)$, that this is a non-stationary Markovian jump process.

Before proceeding to the main results of the paper, we need to introduce some notations.

Let $0=a_{0}<a_{1}<\cdots<a_{k} \leqslant a$ be the time of the process jumps $\{W(c), c \leqslant a\}$, denote by $A_{0}$ the area of the domain enveloped by lines $y=Y(0), y=\frac{x^{2}}{2 b_{n}}, A_{i}$ is the area of the domain enveloped by the lines

$$
y=a_{i-1}\left(x-X\left(a_{i-1}\right)\right)+Y\left(a_{i-1}\right), \quad y=a_{i}\left(x-X\left(a_{i-1}\right)\right)+Y\left(a_{i-1}\right), \quad y=\frac{x^{2}}{2 b_{n}},
$$


for each $1 \leqslant i \leqslant k$.

Let $A[a]$ be the the area of the domain enveloped by the curves

$$
y=a_{k}\left(x-X\left(a_{k}\right)\right)+Y\left(a_{k}\right), \quad y=a\left(x-X\left(a_{k}\right)\right)+Y\left(a_{k}\right), \quad y=\frac{x^{2}}{2 b_{n}} .
$$

If to assume that $A(0, a)=A_{0}+A_{1}+\cdots+A_{k}+A[a]$, then, by from the property of the independence of increments of the Poisson point process, $A(0, a)$ behaves as the sum of a random number of independent random variables, see [13].

As P. Groeneboom noted, to simplify the calculations, $L(x)=1$ can be taken, since all the calculations can easily be transferred to the case of an arbitrary slowly varying function. Then

$$
\begin{aligned}
& \Lambda_{n}^{0}(A)=\left\{\begin{array}{cc}
\frac{\beta}{2 \pi \sqrt{b_{n}}} \int_{A}\left(y-\frac{x^{2}}{2 b_{n}}\right)^{\beta-1} d x d y & \text { as } \quad A \subset R_{n}, \\
0 & \text { as } A \not \subset R_{n} .
\end{array}\right. \\
& b_{n}=n^{\frac{2}{2 \beta+1}}
\end{aligned}
$$

The following theorem holds true.

Theorem 2.1. At $n \rightarrow \infty$, the identity holds

$$
\mathbb{E} A(0, a)=a b_{n} \lambda_{n}^{(1)}, \quad \mathbb{D} A(0, a)=a b_{n}^{\frac{3}{2}} \lambda_{n}^{(1)},
$$

where

$$
\begin{aligned}
& \lambda_{n}^{(1)} \sim C_{1}, \quad \lambda_{n}^{(2)} \rightarrow C_{2}(\beta)+C_{3}(\beta)+C_{4}(\beta), \\
& C_{1}(\beta)=\frac{2 \beta\left(B\left(\frac{1}{2} ; \beta\right)+B\left(\frac{3}{2} ; \beta\right)\right)}{\sqrt{2} \pi(2 \beta+1)}\left(\frac{\sqrt{2} \pi}{B\left(\beta+1 ; \frac{1}{2}\right)}\right)^{\frac{2 \beta+3}{2 \beta+1}} \Gamma\left(\frac{2 \beta+3}{2 \beta+1}\right) .
\end{aligned}
$$

as $n \rightarrow \infty$. Here $t \lambda_{n}^{(1)}$ and $\lambda_{n}^{(2)}$ are constants; they coincide with the corresponding constants given in [2].

Theorem 2.2. As $n \rightarrow \infty$,

$$
\frac{A(0, a)-a b_{n} \lambda_{n}^{(3)}}{\sqrt{a b_{n}^{\frac{3}{2}} \lambda_{n}^{(4)}}} \stackrel{d}{\Rightarrow} N(0,1)
$$

where $\stackrel{d}{\Rightarrow}$ denotes the convergence in distribution and $N(0,1)$ is a standard normally distributed random variable.

\section{Properties of Vertex PROCESSES OF A CONVEX HULL}

In this section we prove a series of preliminary lemmata, which will be employed then in the proof of Theorems 2.1 and 2.2 .

The first lemma provides forms of distributions $W_{n}(a)$ in various situations.

Lemma 3.1. Let

Then

$$
s=y-a x+\frac{a^{2} b_{n}}{2} .
$$

$$
\mathbb{P}\left(W_{n}(0) \in(d x, d y)\right)=\frac{\beta}{2 \pi \sqrt{b_{n}}} \exp \left(-\frac{y^{\beta+\frac{1}{2}}}{\sqrt{2} \pi} B\left(\beta+1 ; \frac{1}{2}\right)\right)\left(y-\frac{x^{2}}{2 b_{n}}\right)^{\beta-1} d x d y,
$$




$$
\begin{aligned}
\mathbb{P}\left(W_{n}(a)\right. & \in(d x, d y))=\frac{\beta}{2 \pi \sqrt{b_{n}}} \exp \left(-\frac{s^{\beta+\frac{1}{2}}}{\sqrt{2} \pi} B\left(\beta+1 ; \frac{1}{2}\right)\right)\left(y-\frac{x^{2}}{2 b_{n}}\right)^{\beta-1} d x d y, \\
\mathbb{P}\left(W_{n}(a)\right. & \left.=W_{n}(0) / W_{n}(0)=(x, y)\right) \\
& =\exp \left(-\frac{1}{2 \pi \sqrt{b_{n}}}\left[\int_{x-a b_{n}}^{\sqrt{2 b_{n} s}}\left(s-\frac{u^{2}}{2 b_{n}}\right)^{\beta} d u-\int_{x}^{\sqrt{2 b_{n} y}}\left(y-\frac{u^{2}}{2 b_{n}}\right)^{\beta} d u\right]\right) .
\end{aligned}
$$

Proof. Let $v=a(u-x)+y$ be a straight line passing through points $(x, y)$ with an angular coefficient $a$ and $D(a, x, y)$ be the domain enveloped by curves $v=a(u-x)+y$ and $v=\frac{u^{2}}{2 b_{n}}$.

It is easy to see that if $u_{1}$ and $u_{2}$ are the roots of equation

$$
\frac{u^{2}}{2 b_{n}}=a(u-x)+y
$$

then $u_{1,2}=a b_{n} \pm \sqrt{2 b_{n} s}$. Let us find $\Lambda_{n}^{0}(D(a, x, y))$; we recall that $\Lambda_{n}^{0}$ was introduced in 2.2 .

We note that

$$
y+a(u-x)-\frac{u^{2}}{2 b_{n}}=y-a x+\frac{a^{2} b_{n}}{2}-\frac{\left(u-a b_{n}\right)^{2}}{2 b_{n}}=s-\frac{\left(u-a b_{n}\right)^{2}}{2 b_{n}}
$$

Then

$$
\begin{aligned}
\Lambda_{n}^{0}(D(a, x, y)) & =\frac{1}{2 \pi \sqrt{b_{n}}} \int_{u_{1}}^{u_{2}}\left[y+a(u-x)-\frac{u^{2}}{2 b_{n}}\right]^{\beta} d u=\frac{1}{2 \pi \sqrt{b_{n}}} \int_{-\sqrt{2 b_{n} s}}^{\sqrt{2 b_{n} s}}\left(s-\frac{u^{2}}{2 b_{n}}\right)^{\beta} d u \\
& =\frac{\sqrt{2} s^{\beta+\frac{1}{2}}}{\pi} \int_{0}^{1}\left(1-u^{2}\right)^{\beta} d u=\frac{s^{\beta+\frac{1}{2}}}{\pi \sqrt{2}} B\left(\beta+1 ; \frac{1}{2}\right) .
\end{aligned}
$$

Let

$$
d=\sqrt{(\Delta x)^{2}+(\Delta y)^{2}}, \quad v=a u+c_{-}, \quad v=a u+c_{+} b
$$

be two lines parallel to $v=a(u-x)+y$ and passing at a distance $d$ from above and below, respectively.

By $D_{d}^{-}(a, x, y)$ and $D_{d}^{+}(a, x, y)$ we denote the domains enveloped respectively by bounded curves

$$
v=a u+c_{-}, \quad v=\frac{u^{2}}{2 b_{n}} \quad \text { and } \quad v=a u+c_{+}, \quad v=\frac{u^{2}}{2 b_{n}} .
$$

Assume that

$$
\Delta_{x, y}=(x, x+\Delta x) \times(y, y+\Delta y) .
$$

It follows from the definition of $W_{n}(a)$ that if the number of points $\bar{\pi}(A)$ in $A$ of inhomogeneous Poisson point processes $\Pi_{n}(\cdot)$ satisfies the inequality

$$
\mathbb{P}\left(W_{n}(a) \in \Delta_{x, y}\right) \leqslant \mathbb{P}\left(\bar{\pi}\left(\Delta_{x, y}\right) \geqslant 1, \bar{\pi}\left(A_{d}^{-}(a, x, y)\right)=0\right) .
$$

On the other hand, it is easy to see that

$$
\mathbb{P}\left(W_{n}(a) \in \Delta_{x, y}\right) \geqslant \mathbb{P}\left(\bar{\pi}\left(\Delta_{x, y}\right)=1, \bar{\pi}\left(D_{d}^{+}(a, x, y)-\Delta_{x, y}\right)=0\right) .
$$

Taking into consideration a Poisson process property, namely, the independence of increments, using inequalities (3.1) and $(3.2)$ as well as $(2.2)$ as $d \rightarrow 0$, we arrive at the first identity of the lemma.

We proceed to the proof of the second identity. It is easy to confirm understand that by the property of the independence of increments, the chain of identities hold:

$$
\mathbb{P}(W(a)=W(0) / W(0)=(x, y))=\mathbb{P}(\bar{\pi}(D(a, x, y)-D(0, x, y))=0 / \pi(D(0, x, y))=0)
$$




$$
\begin{aligned}
& =\mathbb{P}(\bar{\pi}(D(a, x, y)-D(0, x, y))=0) \\
& \left.=\mathbb{P}\left(\bar{\pi}\left(D^{*}(a, x, y)\right)\right)=0\right) \\
& =\exp \left(-\Lambda_{n}\left(D^{*}(a, x, y)\right)\right)
\end{aligned}
$$

where $D^{*}(a, x, y)$ is a set of points in the domain enveloped by lines

$$
v=a(u-x)+y, \quad v=y, \quad v=\frac{u^{2}}{2 b_{n}} .
$$

By the definition of a measure of $\Lambda_{n}(\cdot)$, we obtain

$$
\begin{aligned}
\Lambda_{n}\left(D^{*}(a, x, y)\right) & =\frac{1}{2 \pi \sqrt{b_{n}}}\left\{\int_{x}^{a b_{n}+\sqrt{2 b_{n} s}}\left(s-\frac{\left(u-a b_{n}\right)^{2}}{2 b_{n}}\right)^{\beta} d u-\int_{x}^{\sqrt{2 b_{n} y}}\left(y-\frac{u^{2}}{2 b_{n}}\right)^{\beta} d u\right\} \\
& =\frac{1}{2 \pi \sqrt{b_{n}}}\left\{\int_{x-a b_{n}}^{\sqrt{2 b_{n} s}}\left(s-\frac{u^{2}}{2 b_{n}}\right)^{\beta} d u-\int_{x}^{\sqrt{2 b_{n} y}}\left(y-\frac{u^{2}}{2 b_{n}}\right)^{\beta} d u\right\} .
\end{aligned}
$$

This completes the proof.

Assume that

$$
R_{n}(a)=X_{n}(a)-a b_{n}, \quad S_{n}(a),=Y_{n}(a)-\frac{X_{n}^{2}(a)}{2 b_{n}}+\frac{R_{n}^{2}(a)}{2 b_{n}}, \quad T_{n}(a)=\left(R_{n}(a), S_{n}(a)\right) .
$$

It is obvious that

$$
T_{n}(0)=W_{n}(0) \quad \text { a.s. }
$$

and therefore

$$
\mathbb{P}\left(T_{n}(0) \in(d r, d s)\right)=\mathbb{P}\left(W_{n}(0) \in(d r, d s)\right) .
$$

Lemma 3.2. $T_{n}(a)$ forms a Markovian jump process and

$$
\begin{aligned}
\mathbb{P}\left(T_{n}(0)\right. & \in(d r, d s))=\frac{\beta}{2 \pi \sqrt{b_{n}}} \exp \left\{-\frac{s^{\beta+\frac{1}{2}}}{\sqrt{2} \pi} B\left(\beta+1 ; \frac{1}{2}\right)\right\}\left(s-\frac{r^{2}}{2 b_{n}}\right)^{\beta-1} d r d s, \\
\mathbb{P}\left(T_{n}(a)\right. & \left.=\left(r_{1}, s_{1}\right) / T_{n}(0)=\left(r_{0}, s_{0}\right)\right) \\
& =\exp \left(-\frac{1}{\sqrt{2} \pi}\left[s_{1}^{\beta+\frac{1}{2}} \int_{\frac{r_{1}}{\sqrt{2 b_{n} s_{1}}}}^{1}\left(1-t^{2}\right)^{\beta} d t-s_{0}^{\beta+\frac{1}{2}} \int_{\frac{r_{0}}{\sqrt{2 b_{n} s_{0}}}}^{1}\left(1-t^{2}\right)^{\beta} d t\right],\right.
\end{aligned}
$$

where $r_{1}=r_{0}-a b_{n}, s_{1}=s_{0}-a r_{0}+\frac{a^{2} b_{n}}{2}$, and

$$
\mathbb{P}\left(T_{n}(a) \in\left(d r_{1}, d s_{1}\right) / T_{n}(0)=\left(r_{0}, s_{0}\right)\right)=P\left(T_{n}(a) \in\left(d r_{1}, d s_{1}\right)\right),
$$

if

$$
a b_{n}-\sqrt{2 b_{n} s_{1}}>\sqrt{2 b_{n} s_{0}}
$$

and

$$
\begin{aligned}
& \mathbb{P}\left(T_{n}(a) \in\left(d r_{2}, d s_{2}\right) / T_{n}(0)=\left(r_{1}, s_{1}\right)\right) \\
& =\frac{1}{2 \pi \sqrt{b_{n}}} \exp \left(-\frac{1}{\sqrt{2} \pi}\left(s_{2}^{\beta+\frac{1}{2}} \int_{\frac{s_{1}-s_{2}}{a \sqrt{2 b_{n} s_{2}}}+\frac{a b_{n}}{\sqrt{2 b_{n} s_{2}}}}^{1}\left(1-t^{2}\right)^{\beta} d t\right.\right.
\end{aligned}
$$




$$
\left.\left.-s_{1}^{\beta+\frac{1}{2}} \int_{\frac{s_{1}-s_{2}}{a \sqrt{2 b_{n} s_{1}}}+\frac{a b_{n}}{\sqrt{2 b_{n} s_{1}}}}^{1}\left(1-t^{2}\right)^{\beta} d t\right)\right)\left(s_{2}-\frac{r_{2}^{2}}{2 b_{n}}\right)^{\beta-1} d r_{2} d s_{2}
$$

Here

$$
\begin{aligned}
& \left(r_{i}, s_{i}\right) \in D=\left\{(r, s): s \geqslant \frac{r^{2}}{2 b_{n}}\right\}, \quad a b_{n}-\sqrt{2 b_{n} s_{2}} \leqslant \sqrt{2 b_{n} s_{1}}, \\
& s_{2}+\frac{a^{2} b_{n}}{2}+a r_{2} \geqslant s_{1} \geqslant s_{2}-\frac{a^{2} b_{n}}{2}+a r_{1} .
\end{aligned}
$$

Proof. Statement 1 of the lemma is implied immediately by the definition of $T_{n}(a)$ and Lemma 3.1 .

In what follows, we consider three possible cases regarding the position of the vertex process $W_{n}(a)$.

Case 1: No jumps as the time changes from $a$ to $b$ (Statement 2);

Case 2: There are jumps as the time changes from $a$ to $b$, but the sets $D\left(a, x_{1}, y_{1}\right)$ and $D\left(b, x_{2}, y_{2}\right)$, determining the jump state $W_{n}(\cdot)$ at the time point $a$ and $b$ are disjoint (Statement $3)$;

Case 3: As the time changes from $a$ to $b$, there are jumps and the sets $D\left(a, x_{1}, y_{1}\right)$ and $D\left(b, x_{2}, y_{2}\right)$ determining the jump state $W_{n}(\cdot)$ at the moment of time $a$ and $b$ intersect (Statement 4).

All cases can be treated in a similar way, this is why we deal with Case 1 only. We are going to calculate the probability

$$
\mathbb{P}(a, b)=\mathbb{P}(W(b)=W(a) / W(a)=(x, y)) ;
$$

here the methods from second part of the proof of Lemma 3.1 are used.

We have:

$$
\begin{aligned}
\mathbb{P}(a, b)= & \exp \left(-\frac{1}{2 \pi \sqrt{b_{n}}}\left(\int_{x}^{b b_{n}+\sqrt{2 b_{n} S(b)}}\left(b(u-x)+y-\frac{u^{2}}{2 b_{n}}\right)^{\beta} d u\right.\right. \\
& \left.\left.-\int_{x}^{a b_{n}+\sqrt{2 b_{n} S(a)}}\left(a(u-x)+y-\frac{u^{2}}{2 b_{n}}\right)^{\beta} d u\right)\right),
\end{aligned}
$$

where

$$
S(a)=y-a x+\frac{a^{2} b_{n}}{2}, \quad S(b)=y-b x+\frac{b^{2} b_{n}}{2} .
$$

We let $r(a)=x-a b_{n}, r(b)=x-b b_{n}$, then

$$
a(u-x)+y-\frac{u^{2}}{2 b_{n}}=y-a x+\frac{a^{2} b_{n}}{2}-\frac{\left(u-a b_{n}\right)^{2}}{2 b_{n}}=s(a)-\frac{\left(u-a b_{n}\right)^{2}}{2 b_{n}}
$$

Substituting the variables, we get

$$
\mathbb{P}(a, b)=\exp \left(-\frac{1}{2 \pi \sqrt{b_{n}}}\left(\int_{r(b)}^{\sqrt{2 b_{n} s(b)}}\left(s(b)-\frac{u^{2}}{2 b_{n}}\right)^{\beta} d u-\int_{r(a)}^{\sqrt{2 b_{n} s(a)}}\left(s(a)-\frac{u^{2}}{2 b_{n}}\right)^{\beta} d u\right)\right)
$$




$$
\begin{aligned}
& =\exp \left(-\frac{1}{2 \pi \sqrt{b_{n}}}\left(s(b)^{\beta+\frac{1}{2}} \int_{\frac{r(b)}{\sqrt{2 b_{n} s(b)}}}^{1}\left(1-t^{2}\right)^{\beta} d t-s(a)^{\beta+\frac{1}{2}} \int_{\frac{r(a)}{\sqrt{2 b_{n} s(a)}}}^{1}\left(1-t^{2}\right)^{\beta} d t\right)\right) \\
& =\mathbb{P}(T(b)=(r(b), s(b)) / T(a)=(r(a), s(a))) .
\end{aligned}
$$

Redenoting

$$
\begin{aligned}
r_{0}=x, \quad s_{0}=y, & r_{1}=x-a b_{n}, \\
s_{1}=y-a x+\frac{a^{2} b_{n}}{2}, & r_{2}=x-b b_{n}, \quad s_{2}=y-b x+\frac{b^{2} b_{n}}{2},
\end{aligned}
$$

we obtain

Hence,

$$
r_{2}=r_{1}-(b-a) b_{n}, \quad s_{2}=s_{1}-(b-a) r_{1}+\frac{(b-a)^{2} b_{n}}{2}
$$

$$
\mathbb{P}\left(T(b)=\left(r_{2}, s_{2}\right) / T(a)=\left(r_{1}, s_{1}\right)\right)=\mathbb{P}\left(T(b-a)=\left(r_{2}, s_{2}\right) / T(0)=\left(r_{1}, s_{1}\right)\right)
$$

this proves Statement 2 of the lemma is obtained. The proof is complete.

We consider the following $\sigma$-algebras generated by process $T_{n}(a)$ :

$$
\Im_{n}^{0}=\sigma\left\{T_{n}(c): c \leqslant 0\right\}, \quad \Im_{n}^{a+}=\sigma\left\{T_{n}(c): c \geqslant a\right\} .
$$

Lemma 3.3. For every $A \in \Im_{n}^{0}$ and $B \in \Im_{n}^{a+}$, the inequality

$$
|\mathbb{P}(A \cap B)-\mathbb{P}(A) \mathbb{P}(B)| \leqslant \tau_{n}(a)
$$

holds, where

$$
\tau_{n}(a) \leqslant 4 \exp \left(-\frac{1}{\sqrt{2} \pi}\left(\frac{a^{2} b_{n}}{8}\right)^{\beta+\frac{1}{2}} B\left(\beta+1 ; \frac{1}{2}\right)\right) .
$$

In particular, if

$$
a>\frac{a_{n} \varepsilon_{n}^{*}}{b_{n}}, \quad a_{n}=\sqrt{2 b_{n} \log n}, \quad \varepsilon_{n}^{*}=(\log n)^{-\frac{2 \beta-\delta-1}{2(2 \beta+1)}}, \quad 0<\delta<1,
$$

then

$$
\tau_{n}(a) \leqslant 4 \exp \left(-c(\log n)^{1+\frac{\delta}{3}}\right)
$$

Proof. We introduce events:

$$
G_{1}=\left\{\omega: S_{n}(0) \leqslant \frac{a^{2} b_{n}}{8}\right\}, \quad G_{2}=\left\{\omega: S_{n}(a) \leqslant \frac{a^{2} b_{n}}{8}\right\}, \quad G=G_{1} \cap G_{2} .
$$

Since the sets $G_{1}$ and $G_{2}$ are disjoint, due to the property of the independence of increments, inhomogeneous Poisson point processes for each $A \in G_{1} \in \Im_{n}^{0}$ and $B \in G_{2} \in \Im_{n}^{a+}$ we have

$$
\mathbb{P}(A \cap B / G)=\mathbb{P}(A / G) \mathbb{P}(B / G)
$$

Then it is easy to see that

$$
\begin{aligned}
\mathbb{P}(A \cap B) \geqslant \mathbb{P}(A \cap B \cap G) & =\mathbb{P}(G) \mathbb{P}(A \cap B / G) \\
& =\mathbb{P}(G) \mathbb{P}(A / G) \mathbb{P}(B / G) \\
& =\frac{\mathbb{P}(A \cap G) \mathbb{P}(B \cap G)}{\mathbb{P}(G)} \\
& \geqslant \mathbb{P}(A \cap G) \mathbb{P}(B \cap G) \\
& =(\mathbb{P}(A)-\mathbb{P}(\bar{G}))(\mathbb{P}(B)-\mathbb{P}(\bar{G}))
\end{aligned}
$$




$$
\geqslant \mathbb{P}(A) \mathbb{P}(B)-2 \mathbb{P}(\bar{G}) .
$$

Here $\bar{G}$ is a completement to the event $G$. On the other hand,

$$
\begin{aligned}
\mathbb{P}(A \cap B) \leqslant \mathbb{P}(A \cap B \cap G)+\mathbb{P}(\bar{G}) & =\mathbb{P}(G) \mathbb{P}(A \cap B / G)+\mathbb{P}(\bar{G}) \\
& =\mathbb{P}(G) \mathbb{P}(A / G) \mathbb{P}(B / G)+\mathbb{P}(\bar{G}) \\
& =\frac{\mathbb{P}(A \cap G) \mathbb{P}(B \cap G)}{\mathbb{P}(G)}+\mathbb{P}(\bar{G}) \\
& =\mathbb{P}(A \cap G) \mathbb{P}(B \cap G)+\frac{\mathbb{P}(\bar{G})}{\mathbb{P}(G)} \mathbb{P}(A \cap G) \mathbb{P}(B \cap G)+\mathbb{P}(\bar{G}) \\
& \leqslant \mathbb{P}(A) \mathbb{P}(B)+2 \mathbb{P}(\bar{G}) .
\end{aligned}
$$

Then it is easy to see that

$$
\mathbb{P}(\bar{G})=\mathbb{P}\left(\overline{G_{1}} \cup \overline{G_{2}}\right) \leqslant \mathbb{P}\left(\overline{G_{1}}\right)+\mathbb{P}\left(\overline{G_{2}}\right)=2 \mathbb{P}\left(\overline{G_{1}}\right)=2 \mathbb{P}\left(S_{n}(0) \geqslant \frac{a^{2} b_{n}}{8}\right) .
$$

By last three inequalities we obtain

$$
\begin{aligned}
|\mathbb{P}(A \cap B)-\mathbb{P}(A) \mathbb{P}(B)| & \leqslant 4 \mathbb{P}\left(S_{n}(0) \geqslant \frac{a^{2} b_{n}}{8}\right)=4 \Lambda_{n}^{0}\left(Y \geqslant \frac{a^{2} b_{n}}{8}\right) \\
& =4 \exp \left\{-\frac{1}{\sqrt{2} \pi}\left(\frac{a^{2} b_{n}}{8}\right)^{\beta+\frac{1}{2}} B\left(\beta+1 ; \frac{1}{2}\right)\right\} .
\end{aligned}
$$

Hence, as $a>\frac{a_{n} \varepsilon_{n}^{*}}{b_{n}}$, we have

$$
|\mathbb{P}(A \cap B)-\mathbb{P}(A) \mathbb{P}(B)| \leqslant 4 \exp \left\{-c(\log n)^{1+\frac{\delta}{3}}\right\} .
$$

The proof is complete.

This Lemma implies immediately that

Lemma 3.4. For all $\varepsilon>0, a>0$ the inequality

$$
\sum_{n=1}^{\infty} \tau_{n}^{\varepsilon}(a)<\infty
$$

holds true.

We define

$$
M^{(k)}\left(t ; R^{2}\right)=\frac{\beta}{2 \pi \sqrt{b_{n}}} \int_{r}^{\sqrt{2 b_{n} s}}(u-r)^{k}\left(s-\frac{u^{2}}{2 b_{n}}\right)^{\beta-1} d u \frac{\beta}{2 \pi \sqrt{b_{n}}} \int_{0}^{\sqrt{2 b_{n} s}-r} u^{k}\left(s-\frac{(u+r)^{2}}{2 b_{n}}\right)^{\beta-1} d u
$$

where $t=(r, s)$.

Lemma 3.5. The processes

$$
A(a)-\frac{1}{2} \int_{0}^{a}\left(\sqrt{2 b_{n} s(b)}-r(b)\right)^{2} d b
$$

and

$$
A^{2}(a)-\int_{0}^{a} A(b)\left(\sqrt{2 b_{n} s(b)}-r(b)\right)^{2} d b
$$


are martingales with respect to $\sigma$-algebra

$$
\Im_{a}=\sigma\{T(c)=(r(c), s(c)): 0 \leqslant c \leqslant a\} .
$$

Proof. We first note that according the proof of Lemma 3.2 we have

$$
\mathbb{P}\left(W(a+h) \in\left(d x_{2}, d y_{2}\right) / W(a)=\left(x_{1}, y_{1}\right)\right)=\frac{\beta}{2 \pi \sqrt{b_{n}}}\left(y_{2}-\frac{x_{2}^{2}}{2 b_{n}}\right)^{\beta-1} d x_{2} d y_{2}+o(h) .
$$

Let

$$
\begin{gathered}
D^{(0)}(a, h, r, s)=\left\{(u, v): x \leqslant u \leqslant a b_{n}+\sqrt{2 b_{n} s(a)}, a(u-x)+y \leqslant v \leqslant(a+h)(u-x)+y\right\} ; \\
D^{(1)}(a, h, r, s)=\left\{(u, v): a b_{n}+\sqrt{2 b_{n} s(a)}<u \leqslant(a+h) b_{n}+\sqrt{2 b_{n} s(a+h)},\right. \\
\left.\frac{u^{2}}{2 b_{n}} \leqslant v \leqslant(a+h)(u-x)+y\right\}, \\
D(a, h, r, s)=D^{(0)}(a, h, r, s) \cup D^{(1)}(a, h, r, s),
\end{gathered}
$$

where

$$
s(a)=y-a x+\frac{a^{2} b_{n}}{2}, \quad s(a+h)=y-(a+h) x+\frac{(a+h)^{2} b_{n}}{2} .
$$

It is easy to see that the areas are

$$
\begin{aligned}
& S_{D^{(0)}(a, h, r, s)}=\int_{x}^{a b_{n}+\sqrt{2 b_{n} s}} h(u-x) d u=\frac{h}{2}\left(\sqrt{2 b_{n} s}+a b_{n}-x\right)^{2}=\frac{h}{2}\left(\sqrt{2 b_{n} s}-r\right)^{2}, \\
& S_{D^{(1)}(a, h, r, s)}=\int_{a b_{n}+\sqrt{2 b_{n} s(a)}}^{(a+h) b_{n}+\sqrt{2 b_{n} s(a+h)}}\left((a+h)(u-x)+y-\frac{u^{2}}{2 b_{n}}\right) d u=O\left(h^{2}\right) .
\end{aligned}
$$

Further, for each $k$, for small $h>0$ we get

$\mathbb{E}\left\{A(a, a+h)\right.$, in $D^{(0)}(a, h, r, s)$, there are $k$ vertices $\left.W(\cdot) / W(a)=(x, y)\right\}=O\left(h^{k+1}\right)$,

$\mathbb{E}\left\{A(a, a+h)\right.$, in $D^{(0)}(a, h, r, s)$, there are no vertices $\left.W(\cdot) / W(a)=(x, y)\right\}$

$$
\begin{aligned}
& =S_{D^{(0)}(a, h, r, s)} P\left(\text { in } D^{(0)}(a, h, r, s) \text { no vertices } W(\cdot) / W(a)=(x, y)\right) \\
& =\frac{h}{2}\left(\sqrt{2 b_{n} s}-r\right)^{2} \exp \left\{-\Lambda_{n}(D(a, h, r, s))\right\}+o(h) \\
& =\frac{h}{2}\left(\sqrt{2 b_{n} s}-r\right)^{2}+o(h) .
\end{aligned}
$$

This yield the first statement of the Lemma.

Using (3.4), we obtain

$$
\mathbb{E}\left\{A^{2}(a, a+h) / W(a)=(x, y)\right\}=O\left(h^{2}\right) .
$$

Then, by (3.3) and (3.5), we have

$$
\begin{aligned}
\mathbb{E}\left(A^{2}(0, a+h)-A^{2}(0, a) / W(a)=(x, y)\right)= & \mathbb{E}\left(A^{2}(a, a+h) / W(a)=(x, y)\right) \\
& +2 A(0, a) \mathbb{E}(A(a, a+h) / W(a)=(x, y))+o(h) \\
= & h A(a)\left(\sqrt{2 b_{n} s}-r\right)^{2}+o(h) .
\end{aligned}
$$

The proof is complete. 


\section{Proof of Theorems 2.1 and 2.2}

In this section, the asymptotic behavior of the moments $A(0, a)$ is found as $n \rightarrow \infty$ for a fixed $a$.

Using Lemma 3.5, we have

$$
\begin{aligned}
\mathbb{E} A(0, a) & =\frac{1}{2} \int_{0}^{a} E\left(\sqrt{2 b_{n} s(b)}-r(b)\right)^{2} d b \\
& =\frac{a}{2} \mathbb{E}\left(\sqrt{2 b_{n} s(0)}-r(0)\right)^{2} \\
& =\frac{a \beta}{4 \pi \sqrt{b_{n}}} \int_{0}^{\infty} d s_{0} \int_{-\sqrt{2 b_{n} s_{0}}}^{\sqrt{2 b_{n} s_{0}}}\left(\sqrt{2 b_{n} s_{0}}-r_{0}\right)^{2} \exp \left(-\frac{s_{0}^{\beta+\frac{1}{2}}}{\sqrt{2} \pi} B\left(\beta+1 ; \frac{1}{2}\right)\right) \\
& \left.=\frac{a b_{n} \beta}{\sqrt{2} \pi} \int_{0}^{\infty} s^{\beta+\frac{1}{2}} \exp \left(-\frac{s^{\beta+\frac{1}{2}}}{\sqrt{2} \pi} B\left(\beta+1 ; \frac{r_{0}^{2}}{2 b_{n}}\right)^{\beta-1} d r_{0}\right)\right) d s \int_{-1}^{1}(1-r)^{2}\left(1-r^{2}\right)^{\beta-1} d r \\
& =\frac{a b_{n} \beta}{\sqrt{2} \pi} J_{1} J_{2} .
\end{aligned}
$$

Let us find each factor $J_{1}$ and $J_{2}$. We see that

$$
J_{1}=\int_{0}^{\infty} s^{\beta+\frac{1}{2}} \exp \left\{-\frac{s^{\beta+\frac{1}{2}}}{\sqrt{2} \pi} B\left(\beta+1 ; \frac{1}{2}\right)\right\} d s=\frac{2}{2 \beta+1}\left(\frac{\sqrt{2} \pi}{B\left(\beta+1 ; \frac{1}{2}\right)}\right)^{\frac{2 \beta+3}{2 \beta+1}} \Gamma\left(\frac{2 \beta+3}{2 \beta+1}\right) .
$$

We replace the variable $t=r^{2}$ and in view of the identity

$$
\int_{-1}^{1} r\left(1-r^{2}\right)^{\beta-1} d r=0
$$

we get:

$$
\begin{aligned}
J_{2}= & \int_{-1}^{1}(1-r)^{2}\left(1-r^{2}\right)^{\beta-1} d r=\int_{-1}^{1}\left(1-r^{2}\right)^{\beta-1} d r-2 \int_{-1}^{1} r\left(1-r^{2}\right)^{\beta-1} d r \\
& +\int_{-1}^{1} r^{2}\left(1-r^{2}\right)^{\beta-1} d r=2 \int_{0}^{1}\left(1-r^{2}\right)^{\beta-1} d r+2 \int_{0}^{1} r^{2}\left(1-r^{2}\right)^{\beta-1} d r \\
= & \int_{0}^{1} t^{-\frac{1}{2}}(1-t)^{\beta-1} d t+\int_{0}^{1} t^{\frac{1}{2}}(1-t)^{\beta-1} d t=B\left(\frac{1}{2} ; \beta\right)+B\left(\frac{3}{2} ; \beta\right) .
\end{aligned}
$$

Hence,

$$
\lambda_{n}^{(1)} \sim \frac{2 \beta\left(B\left(\frac{1}{2} ; \beta\right)+B\left(\frac{3}{2} ; \beta\right)\right)}{\sqrt{2} \pi(2 \beta+1)}\left(\frac{\sqrt{2} \pi}{B\left(\beta+1 ; \frac{1}{2}\right)}\right)^{\frac{2 \beta+3}{2 \beta+1}} \Gamma\left(\frac{2 \beta+3}{2 \beta+1}\right) .
$$


It is easy to see that

$$
\begin{aligned}
\mathbb{E} A^{2}(0, a)= & \int_{0}^{a} \mathbb{E} A(b)\left(\sqrt{2 b_{n} s(b)}-r(b)\right)^{2} d b \\
= & \int_{0}^{a} d b \sum_{k=0}^{m} \mathbb{E} A(b-(k+1) h, b-k h)\left(\sqrt{2 b_{n} s(b)}-r(b)\right)^{2} \\
= & \int_{0}^{a} d b \sum_{k=0}^{m} \mathbb{E}\left(\mathbb { E } \left(D^{*}\left(b-(k+1) h, b-k h, x_{0}, y_{0}\right)\right.\right. \\
= & \int_{0}^{a} d b \sum_{k=0}^{m} \mathbb{E}\left(\left(\sqrt{2 b_{n} s(b)}-r(b)\right)^{2}\right. \\
& \left.\cdot \mathbb{E}\left(D^{*}\left(b-(k+1) h, b-k h, x_{0}, y_{0}\right) / W(b-k h)=\left(x_{0}, y_{0}\right)\right)\right)+o(h),
\end{aligned}
$$

where

$$
\begin{aligned}
D^{*}\left(a-h, a, x_{0}, y_{0}\right)= & \left\{(u, v): a b_{n}-\sqrt{2 b_{n} s(a)} \leqslant u \leqslant x_{0},\right. \\
& \left.(a-h)\left(u-x_{0}\right)+y_{0} \leqslant v \leqslant a\left(u-x_{0}\right)+y_{0}\right\} .
\end{aligned}
$$

Then the area of the domain $D^{*}\left(a-h, a, x_{0}, y_{0}\right)$ is

$$
\begin{aligned}
S_{D^{*}\left(a-h, a, x_{0}, y_{0}\right)} & =\int_{a b_{n}-\sqrt{2 b_{n} s(a)}}^{x_{0}}\left((a-h)\left(u-x_{0}\right)+y_{0}-a\left(u-x_{0}\right)-y_{0}\right) d u \\
& =-\int_{a b_{n}-\sqrt{2 b_{n} s(a)}}^{x_{0}} h\left(u-x_{0}\right) d u \\
& =\frac{h}{2}\left(a b_{n}-\sqrt{2 b_{n} s(a)}-x_{0}\right)^{2} \\
& =\frac{h}{2}\left(\sqrt{2 b_{n} s(a)}+r(a)\right)^{2} .
\end{aligned}
$$

Hence,

$$
\begin{aligned}
\mathbb{E} & \left(\left(\sqrt{2 b_{n} s(b)}-r(b)\right)^{2} \mathbb{E}\left(A^{*}(b-(k+1) h, b-k h) / \Im(b-k h, \infty)\right)\right) \\
& =\frac{h}{2} \mathbb{E}\left(\left(\sqrt{2 b_{n} s(b)}-r(b)\right)^{2} \mathbb{E}\left(\left(\sqrt{2 b_{n} s(b-k h)}+r(b-k h)\right)^{2} / \Im(b-k h, \infty)\right)\right) \\
& =\frac{h}{2} \mathbb{E}\left(E\left(\left(\sqrt{2 b_{n} s(b)}-r(b)\right)^{2}\left(\sqrt{2 b_{n} s(b-k h)}+r(b-k h)\right)^{2} / \Im(b-k h, \infty)\right)\right) \\
& =\frac{h}{2} \mathbb{E}\left(\left(\sqrt{2 b_{n} s(b)}-r(b)\right)^{2}\left(\sqrt{2 b_{n} s(b-k h)}+r(b-k h)\right)^{2}\right) .
\end{aligned}
$$


On the other hand, we readily confirm that

$$
\mathbb{E}\left(\sqrt{2 b_{n} s(a)}-r(a)\right)^{2}=\mathbb{E}\left(\sqrt{2 b_{n} s(a)}+r(a)\right)^{2} .
$$

Let $h=b / m$, then from the latter identity (4.1), 4.2) imply that

$$
\begin{aligned}
\mathbb{E} A^{2}(0, a)= & \int_{0}^{a} d b \sum_{k=0}^{m-1} \mathbb{E}\left(\left(\sqrt{2 b_{n} s(b)}-r(b)\right)^{2}\right. \\
& \left.\mathbb{E}\left(A^{*}(b-(k+1) h, b-k h) / \Im(b-k h, \infty)\right)\right)+o(h) \\
= & \frac{1}{2} \int_{0}^{a} d b \sum_{k=0}^{m-1} \mathbb{E}\left(\left(\sqrt{2 b_{n} s(b)}-r(b)\right)^{2}\left(\sqrt{2 b_{n} s(b-k h)}+r(b-k h)\right)^{2}\right) h+o(h) .
\end{aligned}
$$

Hence, as $m \rightarrow \infty$, we get

$$
\mathbb{E} A^{2}(0, a)=\frac{1}{2} \int_{0}^{a} d b \int_{0}^{b} d c \mathbb{E}\left\{\left(\sqrt{2 b_{n} s(b)}-r(b)\right)^{2}\left(\sqrt{2 b_{n} s(c)}+r(c)\right)^{2}\right\}
$$

Then it follows from Lemma 3.5 that

$$
\begin{aligned}
\mathbb{D} A(0, a)= & \frac{1}{2} \int_{0}^{a} d b \int_{0}^{b} d c \mathbb{E}\left(\left(\sqrt{2 b_{n} s(b)}-r(b)\right)^{2}\left(\sqrt{2 b_{n} s(c)}+r(c)\right)^{2}\right) \\
& -\left(\frac{1}{2} \int_{0}^{a} d b \mathbb{E}\left(\sqrt{2 b_{n} s(b)}-r(b)\right)^{2}\right)^{2} \\
= & \frac{1}{2} \int_{0}^{a} d b \int_{0}^{b} d c \mathbb{E}\left(\left(\sqrt{2 b_{n} s(b)}-r(b)\right)^{2}\left(\sqrt{2 b_{n} s(c)}+r(c)\right)^{2}\right) \\
& -\frac{1}{4} \int_{0}^{a} \mathbb{E}\left(\sqrt{2 b_{n} s(b)}-r(b)\right)^{2} d b \\
& \left(\int_{0}^{b} \mathbb{E}\left(\sqrt{2 b_{n} s(c)}+r(c)\right)^{2} d c+\int_{b}^{a} \mathbb{E}\left(\sqrt{2 b_{n} s(c)}+r(c)\right)^{2} d c\right) \\
= & \frac{1}{2} \int_{0}^{a} d b \int_{0}^{b} d c\left(\mathbb{E}\left(\sqrt{2 b_{n} s(b)}-r(b)\right)^{2}\left(\sqrt{2 b_{n} s(c)}+r(c)\right)^{2}\right. \\
= & -\frac{1}{2} \int_{0}^{a} d b \int_{0}^{b} d(b-c)\left(\mathbb{E}\left(\sqrt{2 b_{n} s(b-c)}-r(b-c)\right)^{2}\left(\sqrt{2 b_{n} s(0)}+r(0)\right)^{2}\right. \\
& \left.\mathbb{E}\left(\sqrt{2 b_{n} s(b)}-r(b)\right)^{2} \mathbb{E}\left(\sqrt{2 b_{n} s(c)}+r(c)\right)^{2}\right) \\
& \\
& \\
& \\
&
\end{aligned}
$$


Hence,

$$
\begin{aligned}
\mathbb{D} A(0, a)= & \frac{1}{2} \int_{0}^{a}(a-b) d b\left(\mathbb{E}\left(\sqrt{2 b_{n} s(b)}-r(b)\right)^{2}\left(\sqrt{2 b_{n} s(0)}+r(0)\right)^{2}\right. \\
& \left.-\mathbb{E}\left(\sqrt{2 b_{n} s(b)}-r(b)\right)^{2} \mathbb{E}\left(\sqrt{2 b_{n} s(0)}+r(0)\right)^{2}\right) \\
= & \frac{1}{2} \int_{0}^{a \varepsilon_{n}} \cdots+\frac{1}{2} \int_{a \varepsilon_{n}}^{a} \cdots=: I_{1}+I_{2} .
\end{aligned}
$$

Let $a>\mu_{n}^{(1)} b_{n} / \varepsilon_{n}$, then we let

$$
A=\left\{S(0)<\frac{a^{2} b_{n}}{8}, S(a)<\frac{a^{2} b_{n}}{8}\right\}=G_{1} \cap G_{2}
$$

It is easy to see that for each $\tau>0$

$$
\begin{aligned}
& \mid \mathbb{E}\left(\sqrt{2 b_{n} s(a)}-r(a)\right)^{2}\left(\sqrt{2 b_{n} s(0)}+r(0)\right)^{2} \\
& -\mathbb{E}\left(\sqrt{2 b_{n} s(a)}-r(a)\right)^{2} \mathbb{E}\left(\sqrt{2 b_{n} s(0)}+r(0)\right)^{2} \mid \\
& =O\left(\left(a^{2} b_{n}\right)^{2 \beta+\tau} \exp \left(-C\left(a^{2} b_{n}\right)^{\beta+\frac{1}{2}-\tau}\right)\right) .
\end{aligned}
$$

Hence,

$$
I_{2}=o\left(a b_{n}^{\frac{3}{2}}\right)
$$

We are going to estimate $I_{1}$. Let $a \leqslant \mu_{n}^{(1)} b_{n} / \varepsilon_{n}$. We denote

$$
\begin{aligned}
\mathrm{K}_{1}(a)= & \left\{\left(r_{0}, s_{0}\right) \times\left(r_{1}, s_{1}\right): s_{i}>\frac{r_{i}^{2}}{2 b_{n}}, i=\overline{0,1}, r_{1}=r_{0}-a b_{n}, s_{1}=s_{0}-a r_{1}-\frac{a^{2} b_{n}}{2}\right\}, \\
\mathrm{K}_{2}(a)= & \left\{\left(r_{0}, s_{0}\right) \times\left(r_{1}, s_{1}\right): s_{i}>\frac{r_{i}^{2}}{2 b_{n}}, i=\overline{0,1}, \sqrt{2 b_{n} s_{0}}>a b_{n}-\sqrt{2 b_{n} s_{1}}, s_{1}\right. \\
& \left.+\frac{a^{2} b_{n}}{2}+a r_{1} \geqslant s_{0} \geqslant s_{1}-\frac{a^{2} b_{n}}{2}+a r_{0}, s_{0}-a r_{0}+\frac{a^{2} b_{n}}{2} \geqslant s_{1} \geqslant s_{0}-a r_{1}-\frac{a^{2} b_{n}}{2}\right\}, \\
\mathrm{K}_{3}(a)= & \left\{\left(r_{0}, s_{0}\right) \times\left(r_{1}, s_{1}\right): s_{i}>\frac{r_{i}^{2}}{2 b_{n}}, i=\overline{0,1}, \sqrt{2 b_{n} s_{0}}<a b_{n}-\sqrt{2 b_{n} s_{1}}\right\}, \\
\mathrm{K}_{4}(a)= & \left\{\left(r_{0}, s_{0}\right) \times\left(r_{1}, s_{1}\right): s_{i}>\frac{r_{i}^{2}}{2 b_{n}}, i=\overline{0,1}, \sqrt{2 b_{n} s_{0}} \geqslant a b_{n}-\sqrt{2 b_{n} s_{1}}\right\} .
\end{aligned}
$$

Given $b \leqslant a \varepsilon_{n}$, by the steady-state character of the process $T(a)$ we have

$$
\begin{gathered}
I_{1}=\frac{1}{2} \int_{0}^{a \varepsilon_{n}}(a-b) d b\left(\mathbb{E}\left(\sqrt{2 b_{n} s(b)}-r(b)\right)^{2}\left(\sqrt{2 b_{n} s(0)}+r(0)\right)^{2}\right. \\
\left.-\mathbb{E}\left(\sqrt{2 b_{n} s(b)}-r(b)\right)^{2} \mathbb{E}\left(\sqrt{2 b_{n} s(0)}+r(0)\right)^{2}\right) \\
\sim \frac{a\left(1-\theta \varepsilon_{n}\right)}{2} \int_{0}^{a \varepsilon_{n}} d b\left(\mathbb{E}\left(\sqrt{2 b_{n} s(b)}-r(b)\right)^{2}\left(\sqrt{2 b_{n} s(0)}+r(0)\right)^{2}\right.
\end{gathered}
$$




$$
\begin{gathered}
\left.-\mathbb{E}\left(\sqrt{2 b_{n} s(b)}-r(b)\right)^{2} \mathbb{E}\left(\sqrt{2 b_{n} s(0)}+r(0)\right)^{2}\right) \\
=\frac{a\left(1-\theta \varepsilon_{n}\right)}{2} \int_{0}^{a \varepsilon_{n}}\left(\overline{\mathrm{A}_{1}^{\prime}(b)}+\overline{\mathrm{A}_{2}^{\prime}(b)}-\overline{\mathrm{A}_{3}^{\prime}(b)}\right) d b,
\end{gathered}
$$

where

$$
\begin{aligned}
& \overline{\mathrm{A}_{1}^{\prime}(b)}=\mathbb{E}\left(\left(\sqrt{2 b_{n} s(b)}-r(b)\right)^{2}\left(\sqrt{2 b_{n} s(0)}+r(0)\right)^{2} ;(T(0), T(b)) \in \mathrm{K}_{1}(b)\right), \\
& \overline{\mathrm{A}_{2}^{\prime}(b)}=\mathbb{E}\left(\left(\sqrt{2 b_{n} s(b)}-r(b)\right)^{2}\left(\sqrt{2 b_{n} s(0)}+r(0)\right)^{2} ;(T(0), T(b)) \in \mathrm{K}_{2}(b)\right), \\
& \overline{\mathrm{A}_{3}^{\prime}(b)}=\quad P\left(T(0) \in\left(d r_{0}, d s_{0}\right)\right)\left(\sqrt{2 b_{n} s_{0}}+r_{0}\right)^{2} \\
& \left\{\left(r_{0}, s_{0}\right): s_{0}>\frac{r_{0}^{2}}{2 b_{n}}\right\} \\
& \cdot\left(\int_{\left\{\left(r_{1}, s_{1}\right): s_{1}>\frac{r_{1}^{2}}{2 b_{n}}, \sqrt{2 b s_{0}}>b b_{n}-\sqrt{2 b_{n} s_{1}}\right\}} P\left(T(b) \in\left(d r_{1}, d s_{1}\right)\right)\left(\sqrt{2 b_{n} s_{1}}-r_{1}\right)^{2}\right) .
\end{aligned}
$$

Using Statement 2 of Lemma 3.2 , for sufficiently large $n$ we have

$$
\begin{aligned}
& \overline{\overline{\mathrm{A}_{1}^{\prime}}}=\frac{a\left(1-\theta \varepsilon_{n}\right)}{2} \int_{0}^{a \varepsilon_{n}} \overline{\mathrm{A}_{1}^{\prime}(b)} d b \\
& \sim \frac{\sqrt{2} a b_{n}^{\frac{3}{2}} \beta}{\pi} \int_{0}^{\infty} d b \int_{\left(r_{0}, s_{0}\right) \times\left(r_{1}, s_{1}\right) \in \mathrm{K}_{1}(b)} s_{0} s_{1}\left(1-\frac{r_{1}}{\sqrt{2 b_{n} s_{1}}}\right)^{2}\left(1+\frac{r_{0}}{\sqrt{2 b_{n} s_{0}}}\right)^{2} \\
& \cdot \exp \left(-\frac{1}{\sqrt{2} \pi}\left(s_{1}^{\beta+\frac{1}{2}} \int_{\frac{r_{1}}{\sqrt{2 b_{n} s_{1}}}}^{1}\left(1-t^{2}\right)^{\beta} d t-s_{0}^{\beta+\frac{1}{2}} \int_{\frac{r_{0}}{\sqrt{2 b_{n} s_{0}}}}^{1}\left(1-t^{2}\right)^{\beta} d t\right)\right) \\
& \cdot \exp \left(-\frac{s_{0}^{\beta+\frac{1}{2}}}{\sqrt{2} \pi} B\left(\beta+1 ; \frac{1}{2}\right)\right) d r_{0} d s_{0} \\
& =\frac{\sqrt{2} a b_{n}^{\frac{3}{2}} \beta}{\pi} \int_{0}^{\infty} d s_{0} s_{0}^{\beta+2} \int_{-\sqrt{2 b_{n} s_{0}}}^{\sqrt{2 b_{n} s_{0}}} d\left(\frac{r_{0}}{\sqrt{2 b_{n} s_{0}}}\right) \int_{0}^{\infty} d\left(b \sqrt{\frac{2 b_{n}}{s_{0}}}\right)\left(\frac{s_{1}}{s_{0}}\right)\left(1+\frac{r_{0}}{\sqrt{2 b_{n} s_{0}}}\right)^{2} \\
& \cdot\left(1-\frac{\frac{r_{0}}{\sqrt{2 b_{n} s_{0}}}-b \sqrt{\frac{b_{n}}{2 s_{0}}}}{\sqrt{\frac{s_{1}}{s_{0}}}}\right)^{2} \exp \left(-\frac{s_{0}^{\beta+\frac{1}{2}}}{\sqrt{2} \pi}\left(\left(\frac{s_{1}}{s_{0}}\right)^{\beta+\frac{1}{2}} \int_{\frac{r_{0}}{\sqrt{2 b_{n} s_{0}}-b \sqrt{\frac{b_{n}}{2 s_{0}}}}}^{\sqrt{\frac{s_{1}}{s_{0}}}}\left(1-t^{2}\right)^{\beta} d t\right.\right. \\
& \left.\left.-\int_{\frac{r_{0}}{\sqrt{2 b_{n} s_{0}}}}^{1}\left(1-t^{2}\right)^{\beta} d t\right)\right) \exp \left(-\frac{s_{0}^{\beta+\frac{1}{2}}}{\sqrt{2} \pi} B\left(\beta+1 ; \frac{1}{2}\right)\right)\left(1-\frac{r_{0}^{2}}{2 b_{n} s_{0}}\right)^{\beta-1}
\end{aligned}
$$




$$
\sim C_{2}(\beta) a b_{n}^{\frac{3}{2}} \leqslant C_{1} a b_{n}^{\frac{3}{2}} \int_{0}^{\infty} s_{0}^{\frac{3}{2}} \exp \left(-C_{2} s_{0}^{\beta+\frac{1}{2}}\right) d s_{0} \leqslant C_{3} a b_{n}^{\frac{3}{2}} .
$$

In the same way, for sufficiently large $n$, we obtain

$$
\begin{aligned}
& \overline{\overline{\mathrm{A}_{2}^{\prime}}}=\frac{a\left(1-\theta \varepsilon_{n}\right)}{2} \int_{0}^{a \varepsilon_{n}} \overline{\mathrm{A}_{2}^{\prime}(b)} d b \\
& \sim \frac{\beta^{2} a b_{n}^{2}}{\pi^{2}} \int_{0}^{\infty} d b \int_{0}^{\infty} s_{0}^{2 \beta+1} d s_{0} \int_{-1}^{1} d r_{0}\left(1+r_{0}\right)^{2}\left(1-r_{0}^{2}\right)^{\beta-1} \exp \left(-\frac{s_{0}^{\beta+\frac{1}{2}}}{\sqrt{2} \pi} \mathrm{B}\left(\beta+1 ; \frac{1}{2}\right)\right) \\
& \int_{\left(r_{1}, s_{1}\right) \in \mathrm{K}_{2}^{*}\left(b, r_{0}, s_{0}\right)} d r_{1} d s_{1}\left(1-r_{1}\right)^{2} \exp \left(-\frac{1}{\sqrt{2} \pi}\left(s_{1}^{\beta+\frac{1}{2}} \int_{t^{*}(b)}^{1}\left(1-u^{2}\right)^{\beta} d u\right.\right. \\
& \left.\left.-s_{0}^{\beta+\frac{1}{2}} \int_{t^{*}(b) \sqrt{\frac{s_{1}}{s_{0}}}}^{1}\left(1-u^{2}\right)^{\beta} d u\right)\right)\left(\frac{s_{1}}{s_{0}}\right)^{\beta-1}\left(1-r_{1}^{2}\right)^{\beta-1} \\
& \sim \frac{\sqrt{2} \beta^{2} a b_{n}^{\frac{3}{2}}}{\pi^{2}} \int_{0}^{\infty} s_{0}^{2 \beta+\frac{3}{2}} d s_{0} \int_{-1}^{1} d r_{0}\left(1+r_{0}\right)^{2}\left(1-r_{0}^{2}\right)^{\beta-1} \int_{0}^{\infty} d b^{*} \exp \left(-\frac{s_{0}^{\beta+\frac{1}{2}}}{\sqrt{2} \pi} \mathrm{B}\left(\beta+1 ; \frac{1}{2}\right)\right) \\
& \int_{\left(r_{1}, s_{1}^{*}\right) \in \mathrm{K}_{2}^{* *}\left(b^{*}, r_{0}, s_{0}\right)} d r_{1} d s_{1}\left(1-r_{1}\right)^{2} \exp \left(-\frac{s_{0}^{\beta+\frac{1}{2}}}{\sqrt{2} \pi}\left(\left(s_{1}^{*}\right)^{\beta+\frac{1}{2}} \int_{t^{* *}\left(b^{*}\right)}^{1}\left(1-t^{2}\right)^{\beta} d t\right.\right. \\
& \left.\left.-\int_{t^{* *}\left(b^{*}\right) \sqrt{s_{1}^{*}}}^{1}\left(1-t^{2}\right)^{\beta} d t\right)\right)\left(s_{1}^{*}\right)^{\beta-1}\left(1-r_{1}^{2}\right)^{\beta-1} \sim C_{3}(\beta) a b_{n}^{\frac{3}{2}},
\end{aligned}
$$

where

$$
s_{1}^{*}=\frac{s_{1}}{s_{0}}, \quad b^{*}=\frac{b b_{n}}{\sqrt{2 b_{n} s_{0}}}, \quad t^{* *}\left(b^{*}\right)=\frac{1-s_{1}^{*}}{2 b^{*} \sqrt{s_{1}^{*}}}+\frac{b^{*}}{2 \sqrt{s_{1}^{*}}} .
$$

As in the above relations, for sufficiently large $n$ we have

$$
\begin{aligned}
\overline{\overline{\mathrm{A}_{3}^{\prime}}}= & \frac{a\left(1-\theta \varepsilon_{n}\right)}{2} \int_{0}^{a \varepsilon_{n}} \overline{\mathrm{A}_{3}^{\prime}(b)} d b \\
\sim & \frac{\beta^{2} a b_{n}^{\frac{3}{2}}}{\pi^{2}} \int_{0}^{\infty} d s_{0} \int_{-1}^{1} d r_{0} \int_{0}^{\infty} d b s_{0}^{2 \beta+\frac{3}{2}} \exp \left(-\frac{s_{0}^{\beta+\frac{1}{2}}}{\sqrt{2} \pi} \mathrm{B}\left(\beta+1 ; \frac{1}{2}\right)\right)\left(1-r_{0}^{2}\right)^{\beta-1} \\
& \cdot \int_{\left|r_{1}\right|<1 ; 1>b-\sqrt{s_{1}}} s_{1}^{\beta+\frac{1}{2}} d r_{1} d s_{1} \exp \left(-\frac{s_{0}^{\beta+\frac{1}{2}} s_{1}^{\beta+\frac{1}{2}}}{\sqrt{2} \pi} \mathrm{B}\left(\beta+1 ; \frac{1}{2}\right)\right)\left(1-r_{1}^{2}\right)^{\beta-1} \\
= & C_{4}(\beta) a b_{n}^{\frac{3}{2}} .
\end{aligned}
$$

The proof of Theorem 2.1 is complete.

Theorem 2.2 is implied by Theorem 2.1. Lemma 3.4 and Theorem 17.2.2 in [16]. 


\section{BIBLIOGRAPHY}

1. P. Groeneboom. Limit theorems for convex hulls // Probab. Theor. Relat. Fields. 79:3, 327-368 (1988).

2. H. Carnal. Die konvexe Hülle von $n$ rotationssymmetrisch verteilten Punkten // Z. Wahrscheinlichkeitstheorie Verw. Geb. 15, 168-176 (1970).

3. J.Pardon. Central limit theorems for uniform model random polygons // Theor. Probab. 25:3, 823-833 (2012).

4. A.J. Cabo and P. Groeneboom. Limit theorems for functionals of convex hulls // Probab. Theor. Relat. Fields. 100:1, 31-55 (1994).

5. P. Groeneboom. Convex hulls of uniform samples from a convex polygon // Adv. Appl. Prob. 44:2, 330-342 (2012).

6. C. Buchta. Exact formulae for variances of functionals of convex hulls // Adv. Appl. Prob. 45:4, 917-924 (2013).

7. B. Efron. The convex hull of a random set of points // Biometrika. 52:3, 331-343 (1965).

8. A. Reny and R. Sulanke. Über die konvexe Hülle von zufälling gewahlten Punkten // Z. Wahrscheinlichkeitstheorie. Verw. Geb. 2, 75-84 (1963).

9. T. Hsing. On the asymptotic distribution of the area outside a random convex hull in a disk // Ann. Appl. Prob. 4:2, 478-493 (1994).

10. I. Hueter. The convex hull of a normal sample // Adv. Appl. Prob. 26:4, 855-875 (1994).

11. A.V. Nagaev and I.M. Khamdamov. Limit theorems for functionals of random convex hulls // Preprint of Institute of Mathematics, Academy of Sciences of Uzbekistan. Tashkent. 51 (1991). (in Russian)

12. A.V. Nagaev and I.M. Khamdamov. On the role of extreme summands in sums of independent random variables // Teor. Veroyat. Prim. 47:3, 575-583 (2002). [Theor. Prob. Appl.47:3, 533-541 (2003).]

13. A.V. Nagaev. Some properties of convex hulls generated by homogeneous Poisson point processes in an unbounded convex domain // Ann. Inst. Statist. Math. 47:1, 21-29 (1995).

14. I.M. Khamdamov and T.Kh. Adirov. Martingale properties of vertex functionals generated by Poisson point processes // Doklady Akad. Nauk Uzbekistan. 1, 9-11 (2015). (in Russian)

15. I.M. Khamdamov and T.Kh. Adirov. One of the properties of the convex hull generated by a Poisson point process // Uzbek Math. J. 3, 60-63 (2019).

16. I.A. Ibragimov and Yu.U. Linnik. Independent and stationary sequences of random variables. Nauka, Moscow (1965). [Wolters-Noordhoff Publishing Company, Groningen (1971).]

Isakjan Mamasalievich Khamdamov,

Uzbekistan Academy of Sciences

V.I.Romanovskiy Institute of Mathematics,

Mirzo-Ulugbek 81,700125,

Tashkent, Uzbekistan

Tashkent University of

Information Technologies,

A.Timur street 108,700020,

Tashkent, Uzbekistan

E-mail: isakjan.khamdamov@mail.ru,

khamdamov. isakjan@gmail.com 